\title{
The pathways and conditions of effectively combating the COVID- 19 pandemic in Asia
}

\begin{abstract}
:
The rapid outbreak of COVID-19 epidemic urges to seek advanced solutions to combating the disease to control and or minimize its impacts on humankind and society. We employ a causal mechanism approach and develop a crisp-set Qualitative Comparative Analysis (QCA) to study successful pathways in curbing COVID-19 among 37 Asian countries. Structural variables of GDP per capita, governance, democracy, health access and quality and government response indexes from the real-time Oxford COVID19 Government Response Tracker are included as causal conditions in our QCA model. We identified a total of seven pathways, covering countries in different development stages. Regardless of income levels, we found democracy is important in effectively controlling the pandemic. High democratic scores are characterized in 5 over 7 pathways. Massive testing and comprehensive contact tracing strategies are proved to be effective in containing COVID-19 as shown in two pathways associated with developed and emerging countries. Surprisingly, we found limited impacts of stringent containment measures such as gathering restricted and social distancing. We traced back to the early days of COVID-19 and by performing hierarchical agglomerative cluster analysis, we learned that restrictive containment measures in the early days helped prevent the spread of the pandemic, especially in developing countries. Our empirical results suggest that democracy is still essential in controlling the disease while poor countries can effectively combat COVID-19 with appropriate and timely containment measures.
\end{abstract}

